Article

\title{
The Riemann-Lebesgue Integral of Interval-Valued Multifunctions
}

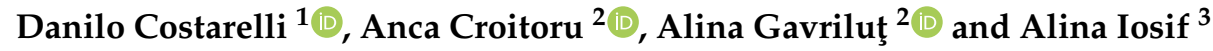 \\ and Anna Rita Sambucini ${ }^{1, * \mathbb{D}}$ \\ 1 Department of Mathematics and Computer Sciences, University of Perugia, 1, Via Vanvitelli, \\ 06123 Perugia, Italy; danilo.costarelli@unipg.it \\ 2 Faculty of Mathematics, University Alexandru Ioan Cuza, Bd. Carol I, No. 11, 700506 Iaşi, Romania; \\ croitoru@uaic.ro (A.C.); gavrilut@uaic.ro (A.G.) \\ 3 Department of Computer Science, Information Technology, Mathematics and Physics, \\ Petroleum-Gas University of Ploieşti, Bd. Bucureşti, No. 39, 100680 Ploieşti, Romania; \\ emilia.iosif@upg-ploiesti.ro \\ * Correspondence: anna.sambucini@unipg.it
}

Received: 10 November 2020; Accepted: 16 December 2020; Published: 20 December 2020

\begin{abstract}
We study Riemann-Lebesgue integrability for interval-valued multifunctions relative to an interval-valued set multifunction. Some classic properties of the $R L$ integral, such as monotonicity, order continuity, bounded variation, convergence are obtained. An application of interval-valued multifunctions to image processing is given for the purpose of illustration; an example is given in case of fractal image coding for image compression, and for edge detection algorithm. In these contexts, the image modelization as an interval valued multifunction is crucial since allows to take into account the presence of quantization errors (such as the so-called round-off error) in the discretization process of a real world analogue visual signal into a digital discrete one.
\end{abstract}

Keywords: Riemann-Lebesgue integral; interval valued (set) multifunction; non-additive set function; image processing

MSC: 28B20; 28C15; 49J53

\section{Introduction}

The theory of multifunctions is an important field of research. Since interval arithmetic, introduced by Moore in [1], it appears a natural option for handling the uncertainty in data and in sensor measurements, particular attention was addressed to the study of interval-valued multifunctions and multimeasures because of their applications in statistics, biology, theory of games, economics, social sciences and software, to keep track of rounding errors in calculations and of uncertainties in the knowledge of the exact values of physical and technical parameters (see for example [2-5]). In fact, since the uncertainty of information could affect an expert's opinion, the ability to consider the uncertainty information during the process could be very important, see for example [2-4,6-11] and the references therein.

However, in some recent papers, interval-valued multifunctions have been applied also to some new directions, involving signal and image processing. Digital images are in fact the result of a discretization of the reality; namely sampled version of a continuous signal. Hence, there are different sources of uncertainty and ambiguity to be considered when performing image processing tasks, see for example [12,13]. For instance, the applications of fractal image coding for image compression $[14,15]$ is one of the topic in which interval-valued multifunctions have been applied. Clearly, image compression 
techniques [16] are very useful in order to speed up the processes of digital image transmission and to improve the efficiency of image storage for high dimensional databases [17]. Further, applications of interval-valued multifunctions to the implementation of edge detection algorithms can also be found (see e.g., $[13,18])$.

In the literature several methods of integration for functions and multifunctions have been studied extending the Riemann and Lebesgue integrals. In this framework a generalization of Riemann sums was given in [19-37] while another generalization is due to Kadets and Tseytlin [38], who introduced the absolute Riemann-Lebesgue $|R L|$ and unconditional Riemann-Lebesgue $R L$ integrability, for Banach valued functions with respect to countably additive measures. They proved that in finite measure space, the Bochner integrability implies $|R L|$ integrability which is stronger than $R L$ integrability that implies Pettis integrability. Regarding this last extension contributions are given also in $[21,23,34,39]$.

In the last decade the study of non-additive set functions and multifunctions has recently received a wide recognition, (see also $[3,9,10,40-46]$ ). In this paper, motivated by the large number of fields in which the interval-valued multifunction can be applied, we introduce a new type of integral of an interval-valued multifunction $G$ with respect to an interval-valued submeasure $M$ with respect to the weak interval order relation introduced in [4] by Guo and Zhang. Although the construction procedure of the integral is similar to the one given in $[34,38,39]$, the integral proposed is a generalization of it since we are concerned with the study of a Riemann-Lebesgue set-valued integrand with respect to an arbitrary interval-valued set function, not necessarily countably additive. So the novelty of this construction concerns not only the codomain of the integrands but also the non-additivity of the measure with respect to which they are integrated. The main results on this subject are Theorem 1 , in which the additivity of the integral is proved even if the pair $(G, M)$ does not satisfy this property; the monotonicity and the order continuity are established in Theorems 2 and 4 and a convergent result given in Theorem 5 .

The paper is organized as follows: in Section 2 the basic concepts and terminology are introduced together with some remarks. In Section 3 we introduce the RL-integral of an interval-valued multifunction with respect to an interval valued subadditive multifunction and we provide a comprehensive treatment of the integration theory together with a comparison with other integrals defined in the same setting (Remark 8). An example of an application in image processing is given in Section 3.1. The applications concerning image processing discussed in the present paper is given for the purpose of illustration and is new. The main reason for which we discuss the above application is to provide examples and justifications of the uses of interval-valued multifunctions to concrete applications in Image Processing. The advantage of using the notion of interval-valued multifunction in signal analysis is that this formalism allows to include in a unique framework possible uncertainty or the noise on the evaluation of an image at any given pixel.

\section{Preliminaries}

Let $S$ be a nonempty at least countable set, $\mathcal{P}(S)$ the family of all subsets of $S$ and $\mathcal{A}$ a $\sigma$-algebra of subsets of $S$. The symbol $\mathbb{R}_{0}^{+}$denotes, as usual, the set of non negative real numbers.

Definition 1 ([34], Definition 2.1).

(i) A finite (countable) partition of $S$ is a finite (countable) family of nonempty sets $P=\left\{A_{i}\right\}_{i=1, \ldots, n}$ $\left(\left\{A_{n}\right\}_{n \in \mathbb{N}}\right) \subset \mathcal{A}$ such that $A_{i} \cap A_{j}=\varnothing, i \neq j$ and $\bigcup_{i=1}^{n} A_{i}=S\left(\bigcup_{n \in \mathbb{N}} A_{n}=S\right)$.

(ii) If $P$ and $P^{\prime}$ are two partitions of $S$, then $P^{\prime}$ is said to be finer than $P$, denoted by $P \leq P^{\prime}$ (or $P^{\prime} \geq P$ ), if every set of $P^{\prime}$ is included in some set of $P$.

(iii) The common refinement of two finite or countable partitions $P=\left\{A_{i}\right\}$ and $P^{\prime}=\left\{B_{j}\right\}$ is the partition $P \wedge P^{\prime}=\left\{A_{i} \cap B_{j}\right\}$.

(iv) A countable tagged partition of $S$ if a family $\left\{\left(B_{n}, s_{n}\right), n \in \mathbb{N}\right\}$ such that $\left(B_{n}\right)_{n}$ is a partition of $S$ and $s_{n} \in B_{n}$ for every $n \in \mathbb{N}$. 
We denote by $\mathcal{P}$ the class of all the countable partitions of $S$ and if $A \in \mathcal{A}$ is fixed, by $\mathcal{P}_{\mathrm{A}}$ we denote the class of all the countable partitions of the set $A$.

Definition 2 ([34], Definition 2.2). Let $m: \mathcal{A} \rightarrow[0,+\infty)$ be a non-negative function, with $m(\varnothing)=0$. $A$ set $A \in \mathcal{A}$ is said to be an atom of $m$ if $m(A)>0$ and for every $B \in \mathcal{A}$, with $B \subset A$, it is $m(B)=0$ or $m(A \backslash B)=0$.

$m$ is said to be:

(i) monotone if $m(A) \leq m(B), \forall A, B \in \mathcal{A}$, with $A \subseteq B$;

(ii) subadditive if $m(A \cup B) \leq m(A)+m(B)$, for every $A, B \in \mathcal{A}$, with $A \cap B=\varnothing$;

(iii) a submeasure (in the sense of Drewnowski [47]) if $m$ is monotone and subadditive;

(iv) $\quad \sigma$-subadditive if $m(A) \leq \sum_{n=0}^{+\infty} m\left(A_{n}\right)$, for every sequence of (pairwise disjoint) sets $\left(A_{n}\right)_{n \in \mathbb{N}} \subset \mathcal{A}$, with $A=\bigcup_{n=0}^{+\infty} A_{n}$.

(v) order-continuous (shortly, o-continuous) if $\lim _{n \rightarrow \infty} m\left(A_{n}\right)=0$, for every decreasing sequence of sets $\left(A_{n}\right)_{n \in \mathbb{N}} \subset \mathcal{A}$, with $A_{n} \searrow \varnothing ;$

(vi) exhaustive if $\lim _{n \rightarrow \infty} m\left(A_{n}\right)=0$, for every sequence of pairwise disjoint sets $\left(A_{n}\right)_{n \in \mathbb{N}} \subset \mathcal{A}$.

(vii) $\quad$ null-additive if $m(A \cup B)=m(A)$, for every $A, B \in \mathcal{A}$, with $m(B)=0$;

Moreover $m$ satisfies property $(\sigma)$ if the ideal of m-zero sets is stable under countable unions (see for example [34], Definition 2.3).

We denote by the symbol $c k(\mathbb{R})$ the family of all non-empty convex compact subsets of $\mathbb{R}$, by convention, $\{0\}=[0,0]$. We consider on $c k(\mathbb{R})$ the Minkowski addition $(A+B:=\{a+b: a \in$ $A, b \in B\}$ ) and the standard multiplication by scalars. $\|A\|:=\sup \{|x|: x \in A\} . d_{H}$ is the Hausdorff distance in $c k(\mathbb{R})$, while $e(A, B)=\sup \{d(x, B), x \in A\}$ and $d_{H}(A, B)=\max \{e(A, B), e(B, A)\}$.

$\left(c k(\mathbb{R}), d_{H}\right)$ is a complete metric space $([48,49])$, but is not a linear space since the subtraction is not well defined.

If $A=[a, b]$ then $\|A\|=\max \{|a|,|b|\}$. Moreover

$$
\begin{aligned}
& d_{H}([a, b],[c, d])=\max \{|a-c|,|b-d|\}, \quad \forall a, b, c, d \in \mathbb{R} \\
& d_{H}([0, a],[0, b])=|b-a| \quad \forall a, b \in \mathbb{R}_{0}^{+} .
\end{aligned}
$$

In the family $c k(\mathbb{R})$ the following operations are also considered, for every $a, b, c, d \in \mathbb{R}$ :

(i) $[a, b] \cdot[c, d]=[a c, b d]$;

(ii) $\quad[a, b] \subseteq[c, d]$ if and only if $c \leq a \leq b \leq d$;

(iii) $\quad[a, b] \preceq[c, d]$ if and only if $a \leq c$ and $b \leq d$; (weak interval order)

(iv) $\quad[a, b] \wedge[c, d]=[\min \{a, c\}, \min \{b, d\}]$;

(v) $\quad[a, b] \vee[c, d]=[\max \{a, c\}, \max \{b, d\}]$.

In general there is no relation between " $\preceq^{\prime \prime}$ (iii) and ${ }^{\prime \prime} \subseteq^{\prime \prime}$ (ii); they only coincide on the subfamily $\{[0, a], a \geq 0\}$. Let $c k\left(\mathbb{R}_{0}^{+}\right):=\{[a, b], a, b \in \mathbb{R}$ and $0 \leq a \leq b\}$.

In this paper we consider $\left(c k\left(\mathbb{R}_{0}^{+}\right), d_{H}, \preceq\right)$, namely the space $c k\left(\mathbb{R}_{0}^{+}\right)$is endowed with the Hausdorff distance and the weak interval order. As a particular case of [20] (Definition 2.1) we have:

Definition 3. Let $\left(a_{n}\right)_{n},\left(b_{n}\right)_{n}$ be two sequences of real numbers so that $0 \leq a_{n} \leq b_{n}, \forall n \in \mathbb{N}$.

The series $\sum_{n=0}^{\infty}\left[a_{n}, b_{n}\right]:=\left\{\sum_{n=0}^{\infty} y_{n}: a_{n} \leq y_{n} \leq b_{n}, \forall n \in \mathbb{N}\right\}$ is called convergent if the sequence of partial sums $S_{n}:=\left[\sum_{k=0}^{n} a_{k}, \sum_{k=0}^{n} b_{k}\right]$ is $d_{H}$-convergent to it.

Remark 1. It is easy to see that $\sum_{n=0}^{\infty}\left[a_{n}, b_{n}\right]=[u, v]$, with $0 \leq u \leq v<\infty$, if and only if $\sum_{n=0}^{\infty} a_{n}=u$ and $\sum_{n=0}^{\infty} b_{n}=v$. 
We recall the following definition for the integrable Banach-valued functions $f: S \rightarrow X$ with respect to non-negative measures given in [38,39]:

Definition 4. A function $f$ is called unconditional Riemann-Lebesgue ( $R L$ ) m-integrable (on $S$ ) if there exists $b \in X$ such that for every $\varepsilon>0$, there exists a countable partition $P_{\varepsilon}$ of $S$, so that for every countable partition $P=\left\{A_{n}\right\}_{n \in \mathbb{N}}$ of $S$ with $P \geq P_{\varepsilon}, f$ is bounded on every $A_{n}$, with $m\left(A_{n}\right)>0$ and for every $t_{n} \in A_{n}, n \in \mathbb{N}$, the series $\sum_{n=0}^{+\infty} f\left(t_{n}\right) m\left(A_{n}\right)$ is unconditional convergent and

$$
\left\|\sum_{n=0}^{+\infty} f\left(t_{n}\right) m\left(A_{n}\right)-b\right\|<\varepsilon
$$

The vector $b$ (necessarily unique) is called the Riemann-Lebesgue m-integral of $f$ on $S$ and it is denoted by $(R L) \int_{S} f d m$. The $R L$ definition of the integrability on a subset $A \in \mathcal{A}$ is given in the classical manner.

Remark 2. We remember that, in the countably additive case, unconditional RL-integrability is stronger than Birkhoff integrability (in the sense of Fremlin), see Ref. [23] and the references therein; while the notion of unconditional Riemann-Lebesgue integrability coincides with Birkhoff's one given in [21] (Definition 1, Proposition 2.6 and note at $p .8$ ).

For the properties of this integral with respect to a submeasure we refer to the results given in [34]. Moreover we have that

Proposition 1. Let $g_{n}: S \rightarrow \mathbb{R}_{0}^{+}$be an increasing sequence of bounded $R L$ integrable function with respect to a submeasure $\mu: \mathcal{A} \rightarrow \mathbb{R}_{0}^{+}$of bounded variation. If there exists a $g: S \rightarrow \mathbb{R}_{0}^{+}$such that

(a) $g_{n} \rightarrow g$ uniformly,

(b) $\sup _{n}(R L) \int_{S} g_{n} d \mu<+\infty$,

then $g$ is $R L$ integrable with respect to $\mu$ and

$$
\lim _{n \rightarrow \infty}(R L) \int_{S} g_{n} d \mu=(R L) \int_{S} g d \mu
$$

Proof. Since $g_{n} \uparrow$, by the monotonicity we have that $(R L) \int_{S} g_{n} d \mu \uparrow$ so $\sup _{n}(R L) \int_{S} g_{n} d \mu=$ $\lim _{n \rightarrow \infty}(R L) \int_{S} g_{n} d \mu=u \in \mathbb{R}_{0}^{+}$. Thanks to uniform convergence $g$ is bounded; let $L>0$ an upper bound for $g$.

Let $\varepsilon>0$ be fixed and consider $k(\varepsilon) \in \mathbb{N}$ be such that

$$
\begin{aligned}
& \left|g(t)-g_{k(\varepsilon)}(t)\right|<\frac{\varepsilon}{3 \mu(S)} \quad \forall t \in S, \quad \text { and } \\
& \left|(R L) \int_{S} g_{k(\varepsilon)} d \mu-u\right|<\frac{\varepsilon}{3} .
\end{aligned}
$$

For every countable partition $P:=\left(A_{n}\right)_{n}$ finer than $P_{\varepsilon / 3, k(\varepsilon)}$ (the one that verifies Definition 4 for $g_{k(\varepsilon)}$ ) and for every $t_{n} \in A_{n}$ we have that $\sum_{n=0}^{+\infty} g\left(t_{n}\right) \mu\left(A_{n}\right)$ converges, since $\mu$ is of bounded variation. 
In fact $g\left(t_{n}\right) \mu\left(A_{n}\right) \leq L \mu\left(A_{n}\right)$ for every $n \in \mathbb{N}$ and, for every $k \in \mathbb{N}$, it is $0 \leq \sum_{n=0}^{k} \mu\left(A_{n}\right) \leq$ $\bar{\mu}(S)$. Moreover

$$
\begin{aligned}
\left|\sum_{n=0}^{+\infty} g\left(t_{n}\right) \mu\left(A_{n}\right)-u\right| & \leq\left|\sum_{n=0}^{+\infty} g\left(t_{n}\right) \mu\left(A_{n}\right)-\sum_{n=0}^{+\infty} g_{k(\varepsilon)}\left(t_{n}\right) \mu\left(A_{n}\right)\right|+ \\
& +\left|\sum_{n=0}^{+\infty} g_{k(\varepsilon)}\left(t_{n}\right) \mu\left(A_{n}\right)-(R L) \int_{S} g_{k(\varepsilon)} d \mu\right|+ \\
& +\left|(R L) \int_{S} g_{k(\varepsilon)} d \mu-u\right| \leq \varepsilon .
\end{aligned}
$$

Remark 3. We can extend Proposition 1 to the bounded sequences $\left(g_{n}\right)_{n}$ that converge $\mu$-almost uniformly on $S$ (namely to the sequences $\left(g_{n}\right)_{n}$ such that for every $\varepsilon>0$ there exists $B(\varepsilon) \in \mathcal{A}$ with $\mu(B(\varepsilon)) \leq \varepsilon$ and $g_{n}$ converges uniformly to $g$ on $S \backslash B(\varepsilon)$ ), if we assume that even $g$ is bounded.

We can proceed in fact in the same way, as in the previous proof, taking $P_{\varepsilon}^{*}:=P_{\varepsilon / 3, k(\varepsilon)} \wedge\{S \backslash B(\varepsilon), B(\varepsilon)\}$ and, for every countable partition $P:=\left(A_{n}\right)_{n}$ finer than $P_{\varepsilon}^{*}$, dividing $\sum_{n=0}^{+\infty} g\left(t_{n}\right) \mu\left(A_{n}\right)$ in two parts: the one relative to $S \backslash B(\varepsilon)$, where the uniform convergence is assumed, and the remining part.

Convergence results in Gould integrability of functions with respect to a submeasure of finite variation are established for instance in [50].

Given two submeasures $\mu_{1}, \mu_{2}: \mathcal{A} \rightarrow \mathbb{R}_{0}^{+}$with $\mu_{1}(A) \leq \mu_{2}(A)$ for every $A \in \mathcal{A}$ let $M: \mathcal{A} \rightarrow$ $c k\left(\mathbb{R}_{0}^{+}\right)$defined by

$$
M(A)=\left[\mu_{1}(A), \mu_{2}(A)\right]
$$

$M$ is called an interval submeasure. For results in this subject see for example [3,43].

Let $M: \mathcal{A} \rightarrow c k\left(\mathbb{R}_{0}^{+}\right)$. We say that $M$ is an interval valued multisubmeasure if

- $M(\varnothing)=\{0\} ;$

- $M(A) \preceq M(B)$ for every $A, B \in \mathcal{A}$ with $A \subseteq B \quad$ (monotonicity);

- $\quad M(A \cup B) \preceq M(A)+M(B)$ for every disjoint sets $A, B \in \mathcal{A}$ (subadditivity).

In literature the multimeasures that satisfy the first two statements are also called set valued fuzzy measures (see for example [4] (Definition 1), [3,11,42-44] and the references therein).

A very interesting case of interval-valued multisubmeasure was given, for the first time, in $[6,8]$ where Dempster and Shefer proposed a mathematical theory of evidence using non additive measures: Belief and Plausibility in such a way for every set $A$ the Belief interval of the set is $[\operatorname{Bel}(A), P l(A)]$. This theory is capable of deriving probabilities for a collection of hypotheses and it allows the system inferencing with the imprecision and uncertainty. If the target space is $c k([0,1])$ it is used for example in decision theory.

We say that $M$ is an additive multimeasure if $M(A \cup B)=M(A)+M(B)$ for every disjoint sets $A, B \in \mathcal{A}$.

If a multimeasure $M$ is countably additive in the Hausdorff metric $d_{H}$, then it is called a $d_{H}$-multimeasure. In this case we have that $\lim _{n \rightarrow \infty} d_{H}\left(\sum_{k=1}^{n} M\left(A_{k}\right), M(A)\right)=0$, for every sequence of pairwice disjoint sets $\left(A_{n}\right)_{n} \subset \mathcal{A}$ such that $\cup_{n} A_{n}=A$.

Remark 4. By Ref. [43] (Remark 3.6) $M(A)=\left[\mu_{1}(A), \mu_{2}(A)\right]$ is a multisubmeasure with respect to $\preceq$ if and only if $\mu_{1}, \mu_{2}$ are submeasures in the sense of Definition 2 (iii). Moreover $M$ is monotone, finitely additive, order-continuous, exhaustive respectively if and only if the set functions $\mu_{1}$ and $\mu_{2}$ are the same (see [40] (Proposition 2.5, Remark 3.3)). 
Definition 5. Let $M: \mathcal{A} \rightarrow c k\left(\mathbb{R}_{0}^{+}\right)$. The variation of $M$ is the set function $\bar{M}: \mathcal{P}(S) \rightarrow[0,+\infty]$ defined by

$$
\bar{M}(E)=\sup \left\{\sum_{i=1}^{n}\left\|M\left(A_{i}\right)\right\|,\left\{A_{i}\right\}_{i=1}^{n} \subset \mathcal{A}, A_{i} \subseteq E, A_{i} \cap A_{j}=\varnothing, i \neq j\right\} .
$$

$M$ is said to be of finite variation if $\bar{M}(S)<\infty$.

Remark 5. We can observe that if $E \in \mathcal{A}$, then in the definition of $\bar{M}$ one may consider the supremum over all finite partitions $\left\{A_{i}\right\}_{i=1}^{n} \in \mathcal{P}_{E}$. If $M$ is finitely additive, then $\bar{M}(A)=M(A)$, for every $A \in \mathcal{A}$.

If $M$ is subadditive (countably subadditive, respectively) of finite variation, then $\bar{M}$ is finitely additive (countably additive, respectively). Finally, if $M(A)=\left[\mu_{1}(A), \mu_{2}(A)\right]$, for every $A \in \mathcal{A}$, then $\bar{M}=\bar{\mu}_{2}$.

\section{3. $R L$ Interval Valued Integral and Its Properties}

In this section, we introduce and study Riemnn-Lebesgue integrability of interval-valued multifunctions with respect to interval-valued set multifunctions, pointing out various properties of this integral. For this, unless stated otherwise, in what follows suppose $S$ is a nonempty set, with card $S \geq \aleph_{0}$ (card $S$ is the cardinality of $S$ ), $\mathcal{A}$ is a $\sigma$-algebra of subsets of $S$.

The multisubmeasure $M$ here considered is an interval-valued one and satisfies (1).

Given $g_{1}, g_{2}: S \rightarrow \mathbb{R}_{0}^{+}$with $g_{1}(s) \leq g_{2}(s)$ for all $s \in S$, let $G: S \rightarrow c k\left(\mathbb{R}_{0}^{+}\right)$be the interval-valued multifunction defined by $G(s)=\left[g_{1}(s), g_{2}(s)\right]$ for every $s \in S$. For every countable tagged partition $\Pi:=\left\{\left(B_{n}, s_{n}\right), n \in \mathbb{N}\right\}$ of $S$ we denote by

$$
\begin{aligned}
\sigma_{G, M}(\Pi) & :=\sum_{n=1}^{\infty} G\left(s_{n}\right) \cdot M\left(B_{n}\right)=\sum_{n=1}^{\infty}\left[g_{1}\left(s_{n}\right) \mu_{1}\left(B_{n}\right), g_{2}\left(s_{n}\right) \mu_{2}\left(B_{n}\right)\right]= \\
& =\left\{\sum_{n=1}^{\infty} y_{n}, y_{n} \in\left[g_{1}\left(s_{n}\right) \mu_{1}\left(B_{n}\right), g_{2}\left(s_{n}\right) \mu_{2}\left(B_{n}\right)\right], n \in \mathbb{N}\right\} .
\end{aligned}
$$

By [20] (Lemma 2.2) the set $\sigma_{G, M}(\Pi)$ is closed and convex in $\mathbb{R}_{0}^{+}$, so it is an interval $\left[u_{G, M}^{(\Pi)}, v_{G, M}^{(\Pi)}\right]$.

Definition 6. A multifunction $G: S \rightarrow c k\left(\mathbb{R}_{0}^{+}\right)$is called Riemann-Lebesgue $R L$ integrable with respect to $M$ (on $S$ ) if there exists $[a, b] \in c k\left(\mathbb{R}_{0}^{+}\right)$such that for every $\varepsilon>0$, there exists a countable partition $P_{\varepsilon}$ of $S$, so that for every tagged partition $P=\left\{\left(A_{n}, t_{n}\right)\right\}_{n \in \mathbb{N}}$ of $S$ with $P \geq P_{\varepsilon}$, the series $\sigma_{G, M}(P)$ is convergent and

$$
d_{H}\left(\sigma_{G, M}(P),[a, b]\right)<\varepsilon .
$$

$[a, b]$ is called the Riemann-Lebesgue integral of $G$ with respect to $M$ and it is denoted

$$
[a, b]=(R L) \int_{S} G d M .
$$

Obviously, if it exists, is unique.

Example 1. Suppose $S=\left\{s_{n} \mid n \in \mathbb{N}\right\}$ is countable, $\left\{s_{n}\right\} \in \mathcal{A}$, for every $n \in \mathbb{N}$, and let $G: S \rightarrow \operatorname{ck}\left(\mathbb{R}_{0}^{+}\right)$be such that the series $\sum_{n=0}^{\infty} g_{i}\left(s_{n}\right) \mu_{i}\left(\left\{t_{n}\right\}\right), i=1,2$ are convergent. Then $G$ is $R L$ integrable with respect to $M$ and

$$
\text { (RL) } \int_{S} G d M=\left[\sum_{n=0}^{\infty} g_{1}\left(s_{n}\right) \mu_{1}\left(\left\{s_{n}\right\}\right), \sum_{n=0}^{\infty} g_{2}\left(s_{n}\right) \mu_{2}\left(\left\{s_{n}\right\}\right)\right] .
$$


Observe moreover that, in this case, the RL-integrability of such $G$ with respect to $M$ implies that the product $G \cdot G$, as defined in $\mathbf{i}$ ), is integrable in the same sense. In particular, if such $G$ is a discrete or countable interval-valued signal, the $(R L) \int_{S} G \cdot G d M$ represents the energy of the signal.

If $M$ is of bounded variation and $G: S \rightarrow c k\left(\mathbb{R}_{0}^{+}\right)$is bounded and such that $G=\{0\} M$-a.e., then, by [34] (Theorem 3.4), $G$ is $M$-integrable and $(R L) \int_{S} G d M=\{0\}$.

From now on we suppose that $G$ is bounded and $\mu_{2}$ is of finite variation.

Proposition 2. An interval multifunction $G=\left[g_{1}, g_{2}\right]$ is $R L$ integrable with respect to $M$ on $S$ if and only if $g_{i}$ are $R L$ integrable with respect to $\mu_{i}, i=1,2$ and

$$
\int_{S} G d M=\left[(R L) \int_{S} g_{1} d \mu_{1},(R L) \int_{S} g_{2} d \mu_{2}\right]
$$

Proof. Suppose that $G=\left[g_{1}, g_{2}\right]$ is $R L$ integrable with respect to $M=\left[\mu_{1}, \mu_{2}\right]$, that means there exists $[a, b] \in c k\left(\mathbb{R}_{0}^{+}\right)$such that for every $\varepsilon>0$, there exists a countable partition $P_{\varepsilon}$ of $S$, so that for every tagged partition $P=\left\{\left(A_{n}, t_{n}\right)\right\}_{n \in \mathbb{N}}$ of $S$ with $P \geq P_{\mathcal{\varepsilon}}$, the series $\sigma_{G, M}(P)$ is convergent and

$$
d_{H}\left(\left[u_{G, M}^{(P)}, v_{G, M}^{(P)}\right],[a, b]\right):=\max \left\{\left|u_{G, M}^{(P)}-a\right|,\left|v_{G, M}^{(P)}-b\right|\right\}<\varepsilon .
$$

By this inequality it follows that

$$
\max \left\{\left|\sum_{n=1}^{\infty} g_{1}\left(t_{n}\right) \mu_{1}\left(A_{n}\right)-a\right|,\left|\sum_{n=1}^{\infty} g_{2}\left(t_{n}\right) \mu_{2}\left(A_{n}\right)-b\right|\right\} \leq \varepsilon, \quad \forall n \in \mathbb{N},
$$

for every tagged partition $P=\left\{\left(A_{n}, t_{n}\right)\right\}_{n \in \mathbb{N}}$ of $S$ with $P \geq P_{\varepsilon}$ and then $g_{i}$ are $R L$ integrable with respect to $\mu_{i}, i=1,2$. Formula (3) follows from the convexity of the $R L$ integral.

For the converse, for every $\varepsilon>0$, let $P_{\varepsilon, g_{i}}, i=1,2$ two countable partitions that verify the definition of $R L$ integrability for $g_{i}, i=1$, 2. Let $P_{\varepsilon}$ be a countable partition of $S$ with $P_{\varepsilon} \geq P_{\varepsilon, g_{1}} \wedge P_{\varepsilon, g_{2}}$. Then, for every $P:=\left\{B_{n}, n \in \mathbb{N}\right\} \geq P_{\varepsilon}$ and for every $t_{n} \in B_{n}$ it is

$$
\left|\sum_{n=0}^{+\infty} g_{i}\left(t_{n}\right) \mu_{i}\left(B_{n}\right)-(R L) \int_{S} g_{i} d \mu_{i}\right|<\varepsilon, \quad i=1,2
$$

Since $g_{i}, i=1,2$ are selections of $G$ this means that

$$
d_{H}\left(\left[u_{G, M}^{(P)}, v_{G, M}^{(P)}\right],\left[(R L) \int_{S} g_{1} d \mu_{1},(R L) \int_{S} g_{2} d \mu_{2}\right]\right) \leq \varepsilon
$$

and then the assertion follows.

Remark 6. By Definition 6 and Proposition 2 we obtain the following definitions for the following cases:

- If $M=\{\mu\}: \mathcal{A} \rightarrow \mathbb{R}_{0}^{+}$is an arbitrary set function and $G=\left[g_{1}, g_{2}\right]$ with $g_{1}(s) \leq g_{2}(s)$ for every $s \in S$ then

$$
\int_{S} G d M=\left[(R L) \int_{S} g_{1} d \mu,(R L) \int_{S} g_{2} d \mu\right]
$$

- If $M=\left[\mu_{1}, \mu_{2}\right]$ as in (1) and $G=\{g\}: S \rightarrow \mathbb{R}_{0}^{+}$then

$$
\int_{S} G d M=\left[(R L) \int_{S} g d \mu_{1},(R L) \int_{S} g d \mu_{2}\right]
$$


Proposition 3. Let $G$ be an interval valued multifuncion. The $R L$ integrability with respect to $M$ is hereditary on subsets $A \in \mathcal{A}$. Moreover $G$ is $R L$ integrable with respect to $M$ on $A$ if and only if $G \chi_{A}$ (where $\chi_{A}$ is the characteristic function of the set $A$ ) is $R L$ integrable with respect to $M$ on $S$. In this case, for every $A \in \mathcal{A}$,

$$
(R L) \int_{A} G d M=(R L) \int_{S} G \chi_{A} d M .
$$

Proof. Assume that $G$ is $R L$ integrable in $S$ with respect to $M$. Let $A \in \mathcal{A}$ and denote by $[a, b]$ the integral of $G$; then, for every $\varepsilon>0$, there exists a countable partition $P_{\varepsilon}$ of $S$, such that, for every finer countable partition $P^{\prime}:=\left\{A_{n}\right\}_{n \in \mathbb{N}}$ and for every $t_{n} \in A_{n}$ it is

$$
d_{H}\left(\sigma_{G, M}\left(P^{\prime}\right),[a, b]\right) \leq \varepsilon
$$

Let $P_{0}$ be a partition such that $P_{0} \geq P_{\varepsilon} \wedge\{A, T \backslash A\}$, and we denote by $P_{A} \subset P_{0}$ the corresponding partition of the set $A$. Let $\Pi_{A}$ be a partition of $A$ finer than $P_{A}$, and extend it with a common partition of $S \backslash A$ in such a way the new partition is finer than $P_{\varepsilon}$.

It is possible to prove that $\sigma_{G, M}\left(\Pi_{A}\right)$ satisfy a Cauchy principle in $c k\left(\mathbb{R}_{0}^{+}\right)$, and so the first claim follows by the completeness of the space. The equality follows from [34] (Theorem 3.2) and Proposition 2.

Remark 7. It is easy to see that, if $G$ is $R L$ integrable with respect to $M$, for every $\alpha \geq 0$ it is:

(a) $\alpha G$ is RL integrable with respect to $M$ and (RL) $\int_{S} \alpha G d M=\alpha(R L) \int_{S} G d M$.

(b) $G$ is $R L$ integrable with respect to $\alpha M$ and $(R L) \int_{S} G d(\alpha M)=\alpha(R L) \int_{S} G d M$.

Theorem 1. If $G$ is an interval valued $R L$ integrable with respect to $M$ multifunction, then $I_{G}: \mathcal{A} \rightarrow c k\left(\mathbb{R}_{0}^{+}\right)$ defined by

$$
I_{G}(A):=(R L) \int_{A} G d M
$$

is a finitely additive multimeasure.

Proof. By Proposition 3 we have that $I_{G}(A) \in c k\left(\mathbb{R}_{0}^{+}\right)$for every $A \in \mathcal{A}$. In order to prove the additivity we can observe that, for every $A, B \in \mathcal{A}$ with $A \cap B=\varnothing$

$$
I_{G}(A \cup B)=(R L) \int_{S} G \chi_{A \cup B} d M=(R L) \int_{S}\left(G \chi_{A}+G \chi_{B}\right) d M .
$$

If we prove that for every pair of interval valued $R L$ integrable with respect to $M$ multifunctions $G_{1}, G_{2}$ we have that

$$
(R L) \int_{S}\left(G_{1}+G_{2}\right) d M=(R L) \int_{S} G_{1} d M+(R L) \int_{S} G_{2} d M
$$

the assertion follows. In order to prove formula (5) let $\varepsilon>0$ be fixed. Since $G_{1}, G_{2}$ are $R L$ integrable with respect to $M$, for every $\varepsilon>0$ there exists a countable partition $P_{\varepsilon} \in \mathcal{P}$ such that for every $P=\left\{A_{n}\right\}_{n \in \mathbb{N}} \geq P_{\varepsilon}$ and every $t_{n} \in A_{n}, n \in \mathbb{N}$, the series $\sigma_{G_{i}, M}(P), i=1,2$ are convergent and

$$
d_{H}\left(\sigma_{G_{i}, M}(P),(R L) \int_{S} G_{i} d M\right)<\frac{\varepsilon}{2}, \quad i=1,2
$$

Then $\sigma_{G_{1}+G_{2}, M}(P)$ is convergent and, by [48] (Proposition 1.17),

$$
d_{H}\left(\sigma_{G_{1}+G_{2}, M}(P),(R L) \int_{S} G_{1} d M+(R L) \int_{S} G_{2} d M\right)<\varepsilon .
$$


So $G_{1}+G_{2}$ is $R L$ integrable with respect to $M$ and formula (5) is satisfied.

Now applying formula (5) with $G_{1}=G \chi_{A}, G_{2}=G \chi_{B}$ to formula (4) we obtain the additivity of $I_{G}$.

The set-valued integral is monotone relative to the order relation " $\preceq "$ and the inclusion one, with respect to the interval-valued integrands.

Proposition 4. If $F, G$ are two $R L$ integrable with respect to $M$ interval valued multifunctions with $F \preceq G$ then, for every $A \in \mathcal{A}, I_{F}(A) \preceq I_{G}(A)$.

Proof. We will prove for $A=S$. Let $F(s):=\left[f_{1}(s), f_{2}(s)\right], G(s)=\left[g_{1}(s), g_{2}(s)\right]$. By the integrability of $F$ and $G$ we have, by Proposition 2

$$
\begin{aligned}
I_{F}(S) & :=(R L) \int_{S} F d M=\left[(R L) \int_{S} f_{1} d \mu_{1},(R L) \int_{S} f_{2} d \mu_{2}\right], \\
I_{G}(S):=(R L) \int_{S} G d M & =\left[(R L) \int_{S} g_{1} d \mu_{1},(R L) \int_{S} g_{2} d \mu_{2}\right] .
\end{aligned}
$$

Since $f_{i}(s) \leq g_{i}(s)$ for all $s \in S$ and $i=1,2$ by [34] (Theorem 3.10) we have that

$$
(R L) \int_{S} f_{1} d \mu_{1} \leq(R L) \int_{S} g_{1} d \mu_{1}, \quad(R L) \int_{S} f_{2} d \mu_{2} \leq(R L) \int_{S} g_{2} d \mu_{2},
$$

and so by the weak interval order, iii), we have that $I_{F}(S) \preceq I_{G}(S)$.

Corollary 1. If $F, G, F \wedge G, F \vee G$ are RL integrable with respect to an interval valued multisubmeasure $M$ then, for every $A \in \mathcal{A}$,

(a) $\quad(R L) \int_{S} F \wedge G d M \preceq I_{F}(A) \wedge I_{G}(A) ;$

(b) $\quad I_{F}(A) \vee I_{G}(A) \preceq(R L) \int_{S} F \vee G d M$.

Proof. Let $F(s)=\left[f_{1}(s), f_{2}(s)\right], G(s)=\left[g_{1}(s), g_{2}(s)\right], h_{*}(s)=\min \left\{f_{1}(s), g_{1}(s)\right\}, h^{*}(s)=$ $\min \left\{f_{2}(s), g_{2}(s)\right\}$. By [34] (Theorem 3.10) $(R L) \int_{S} h_{*} d \mu_{1} \leq\left\{(R L) \int_{S} f_{1} d \mu_{1},(R L) \int_{S} g_{1} d \mu_{1}\right\}$ and an analogous result holds for $(R L) \int_{S} h^{*} d \mu_{2}$. So the result given in 1.a) follows from the definition of $\preceq$ and $\wedge$.

The second statement follows analogously.

Proposition 5. Let $F, G: S \rightarrow c k\left(\mathbb{R}_{0}^{+}\right)$be bounded so that $F, G$ are $R L$ integrable with respect to $M$. If $F \subseteq G$, then $I_{F}(A) \subseteq I_{G}(A)$ for all $A \in \mathcal{A}$.

Proof. As before we will prove for $S$. Let $\varepsilon>0$ be arbitrary. Since $F, G$ are $R L$ integrable with respect to $M$, there exists a countable partition $\Pi_{\varepsilon}$ of $S$ so that for every other countable partition $\Pi=\left\{B_{n}\right\}_{n \in \mathbb{N}} \in \mathcal{P}$, with $\Pi \geq \Pi_{\varepsilon}$ and every choise of points $s_{n} \in B_{n}, n \in \mathbb{N}$, the series

$$
\sum_{n=0}^{\infty} F\left(s_{n}\right) \cdot M\left(B_{n}\right), \quad \sum_{n=0}^{\infty} G\left(s_{n}\right) \cdot M\left(B_{n}\right)
$$

are convergent and

$$
d_{H}\left(I_{F}(S), \sum_{n=0}^{\infty} F\left(s_{n}\right) \cdot M\left(B_{n}\right)\right)<\frac{\varepsilon}{3} ; \quad d_{H}\left(I_{G}(S), \sum_{n=0}^{\infty} G\left(s_{n}\right) \cdot M\left(B_{n}\right)\right)<\frac{\varepsilon}{3} .
$$


Then, by the triangular property of the eccess $e$,

$$
\begin{aligned}
e\left(I_{F}(S), I_{G}(S)\right) & \leq d_{H}\left(I_{F}(S), \sum_{n=0}^{\infty} F\left(s_{n}\right) \cdot M\left(B_{n}\right)\right)+e\left(\sum_{n=0}^{\infty} F\left(s_{n}\right) \cdot M\left(B_{n}\right), \sum_{n=0}^{\infty} G\left(s_{n}\right) \cdot M\left(B_{n}\right)\right)+ \\
& +d_{H}\left(\sum_{n=0}^{\infty} G\left(s_{n}\right) \cdot M\left(B_{n}\right), I_{G}(S)\right)<\frac{2 \varepsilon}{3}+e\left(\sum_{n=0}^{\infty} F\left(s_{n}\right) \cdot M\left(B_{n}\right), \sum_{n=0}^{\infty} G\left(s_{n}\right) \cdot M\left(B_{n}\right)\right) .
\end{aligned}
$$

Since the series $\sum_{n=0}^{\infty} F\left(s_{n}\right) \cdot M\left(B_{n}\right)$ and $\sum_{n=0}^{\infty} G\left(s_{n}\right) \cdot M\left(B_{n}\right)$ are convergent in $c k\left(\mathbb{R}_{0}^{+}\right)$, and, by hypothesis, $\sum_{n=0}^{\infty} F\left(s_{n}\right) \cdot M\left(B_{n}\right) \subseteq \sum_{n=0}^{\infty} G\left(s_{n}\right) \cdot M\left(B_{n}\right)$, then

$$
e\left(\sum_{n=0}^{\infty} F\left(s_{n}\right) \cdot M\left(B_{n}\right), \sum_{n=0}^{\infty} G\left(s_{n}\right) \cdot M\left(B_{n}\right)\right)=0
$$

Consequently, from the arbitrariety of $\varepsilon>0, e\left(I_{F}(S), I_{G}(S)\right)=0$, which implies $I_{F}(S) \subseteq I_{G}(S)$.

We can observe moreover that

Proposition 6. If $G$ is bounded and $R L$ integrable with respect to $M$, with $M$ of bounded variation, then

(a) $\quad\left\|I_{G}(S)\right\|=(R L) \int_{S} g_{2} d \mu_{2}=(R L) \int_{S}\|G\| d\|M\|$.

(b)

$$
\begin{aligned}
\bar{I}_{G}(S) & =\sup \left\{\sum_{i=1}^{n}\left|I_{G}\left(A_{i}\right)\right|,\left\{A_{i}, i=1, \ldots, n\right\} \in \mathcal{P}\right\}= \\
& =\sup \left\{\sum_{i=1}^{n}(R L) \int_{A_{i}} g_{2} d \mu_{2},\left\{A_{i}, i=1, \ldots, n\right\} \in \mathcal{P}\right\}=(R L) \int_{S} g_{2} d \mu_{2} .
\end{aligned}
$$

Proof. It is a consequence of the properties of $d_{H}$ and [34] (Proposition 3.3, Theorem 3.5).

Proposition 7. Let $G: S \rightarrow c k\left(\mathbb{R}_{0}^{+}\right)$be a bounded multifunction such that $G$ is $R L$ integrable with respect to $M$ on every set $A \in \mathcal{A}$.

(a) If $M$ is of bounded variation, then $I_{G} \ll \bar{M}$ (in the $\varepsilon-\delta$ sense) and $I_{G}$ is of finite variation.

(b) If moreover $M$ is o-continuous (exhaustive respectively), then $I_{G}$ is also o-continuous (exhaustive respectively).

Proof. The statements easily follow by Proposition 6.

Moreover

Theorem 2. Let $G: S \rightarrow c k\left(\mathbb{R}_{0}^{+}\right)$be a multifunction such that $G$ is $R L$ integrable with respect to $M$ on every set $A \in \mathcal{A}$. The following statements hold:

(a) If $M$ is monotone, then $I_{G}$ is monotone too.

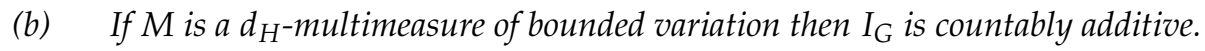

Proof. Let $A, B \in \mathcal{A}$ with $A \subseteq B$. By monotonicity $\mu_{i}(A) \leq \mu_{i}(B)$ for $i=1,2$. We divide $B$ in $A, B \backslash A$ and we apply [34] (Theorem 3.2, Corollary 3.6). The conclusion follows by (iii).

Since $M$ is a $d_{H}$-multimeasure, then $\bar{M}$ is countably additive too and o-continuous. Applying Proposition $7 I_{G}$ is o-continuous too. Let $\left(A_{n}\right)_{n \in \mathbb{N}} \subset \mathcal{A}$ be an arbitrary sequence of pairwise 
disjoint sets, with $\bigcup_{n=1}^{\infty} A_{n}=A \in \mathcal{A}$. We denote by $B_{n}$ the set $B_{n}:=\bigcup_{k=n+1}^{\infty} A_{k}$. Since $B_{n} \searrow \varnothing$, then $\lim _{n \rightarrow \infty}\left\|I_{G}\left(B_{n}\right)\right\|=0$. Since $I_{G}$ is finitely additive, we have

$$
\lim _{n \rightarrow \infty} d_{H}\left(I_{G}(A), \sum_{k=1}^{n} I_{G}\left(A_{k}\right)\right)=\lim _{n \rightarrow \infty} d_{H}\left(\sum_{k=1}^{n} I_{G}\left(A_{k}\right)+I_{G}\left(B_{n}\right), \sum_{k=1}^{n} I_{G}\left(A_{k}\right)\right) \leq \lim _{n \rightarrow \infty}\left\|I_{G}\left(B_{n}\right)\right\|=0
$$

which ensures that $I_{G}$ is a $d_{H}$-multimeasure.

Proceeding as in to the proof of the formula (5) and applying [34] (Theorem 3.8) we obtain the following result:

Proposition 8. Let be $M_{1}, M_{2}: \mathcal{A} \rightarrow c k\left(\mathbb{R}_{0}^{+}\right)$, with $M_{1}(\varnothing)=M_{2}(\varnothing)=\{0\}$ and suppose $G: S \rightarrow c k\left(\mathbb{R}_{0}^{+}\right)$ is $R L$ integrable with respect to both $M_{1}$ and $M_{2}$. If $M: \mathcal{A} \rightarrow c k\left(\mathbb{R}_{0}^{+}\right)$is the interval-valued multisubmeasure defined by $M(A)=M_{1}(A)+M_{2}(A)$, for every $A \in \mathcal{A}$, then $G$ is $R L$ integrable with respect to $M$ and

$$
(R L) \int_{S} G d\left(M_{1}+M_{2}\right)=(R L) \int_{S} G d M_{1}+(R L) \int_{S} G d M_{2} .
$$

Theorem 3. Let $M$ be of bounded variation and $F, G: T \rightarrow c k\left(\mathbb{R}_{0}^{+}\right)$be bounded interval-valued multifunctions. If $F, G$ are $R L$ integrable with respect to $M$, then

$$
d_{H}\left((R L) \int_{S} F d M,(R L) \int_{S} G d M\right) \leq \sup _{s \in S} d_{H}(F(s), G(s)) \cdot \bar{M}(S) .
$$

Proof. Since $F, G$ are $M$-integrable then $f_{1}, g_{1}$ are $\mu_{1}$-integrable and $f_{2}, g_{2}$ are $\mu_{2}$-integrable functions. According to [34] (Theorem 3.9), we have for $i=1,2$,

$$
\left|(R L) \int_{S} f_{i} d \mu_{i}-(R L) \int_{S} g_{i} d \mu_{i}\right| \leq \sup _{s \in S}\left|f_{i}(s)-g_{i}(s)\right| \bar{\mu}_{i}(S) .
$$

Therefore, by (6) and Remark 5, it follows

$$
\begin{aligned}
d_{H}\left((R L) \int_{S} F d M,(R L) \int_{S} G d M\right) & =\max \left\{\left|(R L) \int_{S} f_{1} d \mu_{1}-(R L) \int_{S} g_{1} d \mu_{1}\right|,\left|(R L) \int_{S} f_{2} d \mu_{2}-(R L) \int_{S} g_{2} d \mu_{2}\right|\right\} \\
& \leq \max \left\{\sup _{s \in S}\left|f_{1}(s)-g_{1}(s)\right| \overline{\mu_{1}}(S), \sup _{s \in S}\left|f_{2}(s)-g_{2}(s)\right| \overline{\mu_{2}}(S)\right\} \leq \\
& \leq \max \left\{\sup _{s \in S}\left|f_{1}(s)-g_{1}(s)\right|, \sup _{s \in S}\left|f_{2}(s)-g_{2}(s)\right|\right\} \overline{\mu_{2}}(S) \leq \\
& =\sup _{s \in S} d_{H}(F(s), G(s)) \bar{M}(S) .
\end{aligned}
$$

Theorem 4. Let $M_{1}, M_{2}: \mathcal{A} \rightarrow c k\left(\mathbb{R}_{0}^{+}\right)$and $G: S \rightarrow c k\left(\mathbb{R}_{0}^{+}\right)$be $R L$ integrable with respect to both $M_{1}$ and $M_{2}$. Then

(a) If $M_{1} \preceq M_{2}$, then (RL) $\int_{S} G d M_{1} \preceq(R L) \int_{S} G d M_{2}$.

(b) If $M_{1} \subseteq M_{2}$, then (RL) $\int_{S} G d M_{1} \subseteq(R L) \int_{S} G d M_{2}$.

Proof. Let $M_{1}:=\left[\mu_{*}, \mu^{*}\right]$ and $M_{2}:=\left[v_{*}, v^{*}\right]$. Both the results are consequences of Theorem 2 and [34] (Theorem 3.11). It is enough to observe that if $M_{1} \preceq M_{2}$ then $\mu_{*} \leq v_{*}$ and $\mu^{*} \leq v^{*}$, while if $M_{1} \subseteq M_{2}$ then $v_{*} \leq \mu_{*} \leq \mu^{*} \leq v^{*}$. 
As a particular case of Theorem 4 and Corollary 1 we have that for every $G$ which is $R L$ integrable with respect to both positive submeasures $\mu_{1}$ and $\mu_{2}$ then

$$
(R L) \int_{S} G d\left(\mu_{1} \wedge \mu_{2}\right) \preceq(R L) \int_{S} G d \mu_{1} \wedge(R L) \int_{S} G d \mu_{2} .
$$

Moreover a convergence result can be obtained using Proposition 1.

Theorem 5. Let $G_{n}=\left[g_{1}^{(n)}, g_{2}^{(n)}\right]$ be a sequence of bounded $R L$-integrable interval valued multifunction with respect to $M=\left[\mu_{1}, \mu_{2}\right]$ such that $G_{n} \preceq G_{n+1}$ for every $n \in \mathbb{N}$. If $M$ is of bounded variation and there exists a function $G=\left[g_{1}, g_{2}\right]$ such that:

(a) $d_{H}\left(G_{n}, G\right) \rightarrow 0$ uniformly;

(b) $\sup _{n}\left\|(R L) \int_{S} G_{n} d M\right\|<+\infty$,

then $G$ is $R L$-integrable with respect to $M$ and

$$
\lim _{n \rightarrow \infty} d_{H}\left((R L) \int_{S} G_{n} d M,(R L) \int_{S} G d M\right)=0 .
$$

Proof. Since $G_{n} \preceq G_{n+1}$ we have that $g_{i}^{(n)} \uparrow$ for $i=1,2$, this is a consequence of Proposition 4 and Definition 6. By $d_{H}\left(G_{n}, G\right) \rightarrow 0$ uniformly we have that $\max \left\{\left|g_{i}^{(n)}-g_{i}\right|, i=1,2\right\}$ converges uniformly to zero. We can use now Proposition 1 and we obtain

$$
\lim _{n \rightarrow \infty}(R L) \int_{S} g_{i}^{(n)} d \mu_{i}=(R L) \int_{S} g_{i} d \mu_{i}, \quad i=1,2
$$

For every $\varepsilon>0$ let $k(\varepsilon) \in \mathbb{N}$ be such that

$$
d_{H}\left(G(t), G_{k(\varepsilon)}(t)\right)<\varepsilon \forall t \in S, \quad \text { and } \quad\left|(R L) \int_{S} g_{i}^{(k(\varepsilon))} d \mu_{i}-(R L) \int_{S} g_{i} d \mu_{i}\right|<\varepsilon, i=1,2 .
$$

So,

$$
d_{H}\left((R L) \int_{S} G_{k(\varepsilon)} d M,\left[(R L) \int_{S} g_{1} d \mu_{1},(R L) \int_{S} g_{2} d \mu_{2}\right]\right) \leq \varepsilon .
$$

Let $P_{\varepsilon}$ be the countable partition of $S$ given by $\bigwedge_{i=1,2} P_{\varepsilon, i}$, (the ones that verify Definition 4 for $g_{i}^{k(\varepsilon)}, i=1,2$ respectively). Then, for every countable partition $P=\left\{A_{n}\right\}_{n \in \mathbb{N}}$ of $S$ with $P \geq P_{\varepsilon}$ and for every $t_{n} \in A_{n}$ the series $\sigma_{G, M}(P)$ is convergent and

$$
\begin{aligned}
d_{H}\left(\sigma_{G, M}(P),\left[(R L) \int_{S} g_{1} d \mu_{1},(R L) \int_{S} g_{2} d \mu_{2}\right]\right) & <d_{H}\left(\sigma_{G, M}(P), \sigma_{G_{k(\varepsilon)}, M}(P)\right)+ \\
& +d_{H}\left(\sigma_{G_{k(\varepsilon)}, M}(P),(R L) \int_{S} G_{k(\varepsilon)} d M\right)+ \\
& +d_{H}\left((R L) \int_{S} G_{k(\varepsilon)} d M,\left[(R L) \int_{S} g_{1} d \mu_{1},(R L) \int_{S} g_{2} d \mu_{2}\right]\right) .
\end{aligned}
$$

From previous inequalities and by the arbitrariety of $\varepsilon$ the $R L$-integrability of $G$ follows.

Remark 8. Since this research starts from the papers [34,43], this part ends with a comparison between the two types of integral considered: the RL integral with the Gould one given in [43] (Definition 4.7).

If the interval-valued multifunction $F$ is bounded and $\mu_{2}$ is of finite variation then, analogously to Proposition 2 it is, by [43] (Proposition 4.9),

$$
\text { (G) } \int_{S} F d M=\left[(G) \int_{S} f_{1} d \mu_{1},(G) \int_{S} f_{2} d \mu_{2}\right] .
$$


So, the two kinds of integral coincide on bounded interval-valued multifunctions with values in ck $\left(\mathbb{R}_{0}^{+}\right)$when $\mu_{i}, i=1,2$ are complete countably additive measures by [34] (Proposition 4.5) or $\mu_{i}, i=1,2$ are monotone, countably -subadditive by [34] (Theorem 4.7).

Without countable additivity the equivalence does not hold; an example can be constructed using [34] (Example 4.6). In the general case only partial results can be obtained on atoms when $\mu_{i}, i=1,2$ are monotone, null additive and satisfy property $(\sigma)$ : the proof follows from [34] (Theorem 4.8).

Accordingly with the comparison between Gould and Birkoff integrals given in [28] we have that Birkhoff, Gould, RL integrals of the bounded single valued functions agree in the countably additive case, see [28] (Theorem 3.10), while in [43] (Remark 5.5) an analogous comparison is given with the Choquet integral.

A comparison between simple Birkhoff and RL integrabilities, introduced in [23,28], in this non additive setting can be obtained using [34] (Theorem 4.2).

Finally we would like to observe that the Rådström's embedding tell us that $\left(c k(X), d_{H}, \subseteq\right)$, when $X$ finite dimensional, is a near vector space with 0 element and order unit $B_{X}$. In this case, using [51] (Theorem 5.1), it is a near vector space (see [51] (Definition 2.1) for its definition) that could be embedded, for example, in $\ell_{\infty}$ or in $C(\Omega)$ with $\Omega$ compact and Hausdorff in such a way the embedding is an isometric isomorphism which takes into account the ordering on the hyperspace.

If we consider instead $\left(c k\left(\mathbb{R}_{0}^{+}\right), d_{H}, \preceq\right)$, since in general there is no relation between " $\preceq$ " and " $\subseteq$ " the Rådström embedding provide only the integrability of the interval-valued functions and does not take the weak interval order into account. For this reason we preferred to give the the construction of the $R L$ integral and the proofs, both related to $\preceq$, independently of the Rådström's embedding.

\subsection{Applications of Interval Valued Multifunctions}

Now, in order to explain what could be the benefits of this approach we give an example of an application of interval valued multifunctions on interval valued multisubmeasure in image processing. In fact a signal can be modeled as an interval-valued multifunction as in [12]. In fact, when the value of the points can not be assigned with precision, it might be preferable to use a measure-based approach.

The advantage of using the notion of interval-valued multifunction in signal analysis is that this formalism allows to include in a unique framework possible uncertainty or the noise on the value of a point.

This situation usually occurs in signal and image processing when images are derived by a measure process, as happens for instance for biomedical images (in CT images, MR images, etc), and in several other applied sciences. In particular, we can apply this representation to a digital image in such a way:

Example 2. To each pixel (or to a set of pixels) of the image is associated an interval which measures the round-off error which is that committed on the detection on the signal due by the tolerances and by the limits on computational accuracy of the measurements tools ([52]).

When we consider subsets of pixels we are taking into account the so-called time-jitter error, i.e., the error that occur in the measure of a given signal when the sampling values can not be matched exactly at the theoretical node but just in a neighborhood of it (see, e.g., [53]).

In this sense, if $I=\left(m_{i, j}\right)$ is the matrix associated to a $n \times m$ static, gray-scale image, we can consider the space $\mathcal{S}:=(0, n] \times(0, m] \subset \mathbb{R}^{2}$, and hence the interval-valued multifunction $U_{I}: \mathcal{S} \rightarrow \mathcal{K}_{C}^{+}$corresponding to $I$, will be given by:

$$
U_{I}(x):=\left[u_{1}(x), u_{2}(x)\right], \quad x \in \mathcal{S} .
$$

The model of a digital image by an interval-valued multifunction as $U_{I}$, and obtained by a certain discretization (algorithm) of an analogue image, allows to control the round-off error in the sense that, the true value assumed by original signal at the pixel $x$ belongs to the interval $\left[u_{1}(x), u_{2}(x)\right]$, in fact providing a lower and an upper bound on the possible oscillations of the sampled image. 
For example, in fractal image coding, the functions $u_{1}$ and $u_{2}$ represent respectively the lower and upper contraction maps of an image, which take into account of the round-off error in the contraction procedure, and can be chosen as follows:

$$
u_{1}(x):=\alpha_{1} u(x)+\beta_{1}(x), \quad u_{2}(x):=\alpha_{2} u(x)+\beta_{2}(x), \quad x \in \mathcal{S},
$$

where $\alpha_{i}, i=1,2$, are suitable integer scaling parameters, $\beta_{i}: \mathcal{S} \rightarrow \mathbb{N}, i=1,2$, are suitable functions, and $u: \mathcal{S} \rightarrow \mathbb{N}$ is the continuous model associated to the starting image I. The functions $u_{1}$ and $u_{2}$ provide for each pixel the interval containing the true value of the compressed image.

In particular, in the algorithm considered in [15], the functions $u_{1}$ and $u_{2}$ are piecewise constant, and for a starting image of $225 \times 225$ pixel size, they have been defined as follows:

$$
U_{I}(x)=\left[u_{1}(x), u_{2}(x)\right]=[u(x)-\beta(x), u(x)+\beta(x)], \quad x \in \mathcal{S},
$$

where:

$$
u(x):=m_{i, j}, \quad x \in(i-1, i] \times(j-1, j], \quad i, j=1, \ldots, 225,
$$

and

$$
\beta(x):= \begin{cases}0, & x \in(0,115] \times(0,115], \\ 40, & x \in(115,225] \times(115,225], \\ 20, & \text { otherwise. }\end{cases}
$$

As an example we use the interval-valued multifunction (7) to operate with the well-known image of "Baboon" given in Figure 1 (left); the images generated by $u_{1}$ and $u_{2}$ using the function $\beta$ defined in (8) are given in Figure 1 (center and right).
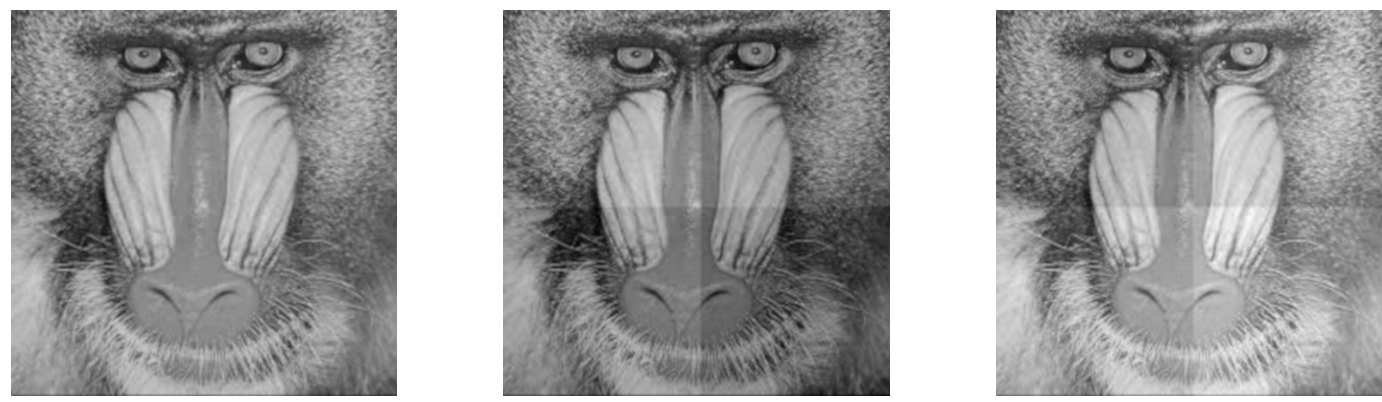

Figure 1. Baboon (left); The images generated by $u_{1}$ (center) and $u_{2}$ (right) using the interval valued multifunction (7), with $\beta$ defined in (8).

Here, also numerical truncation have been taken into account, in order to maintain the values of the pixels in the (integer) gray scale $[0,255]$.

For other examples of functions $u_{1}$ and $u_{2}$, see, e.g., [13,54]. For instance, in [13] the image representation by multifunctions is used for the implementation of edge detection algorithms, and in this case the corresponding functions $u_{1}$ and $u_{2}$ are:

$$
u_{1}(x):=\max \left\{0, \min _{x^{\prime} \in n(x)}\left\{I\left(x^{\prime}\right)-1\right\}\right\}, \quad u_{2}(x):=\min \left\{255, \max _{x^{\prime} \in n(x)}\left\{I\left(x^{\prime}\right)+1\right\}\right\},
$$

where $I(x)$ represents the value of a pixel at a position $x \in \mathcal{S}$, while $n(x)$ denotes any set of $3 \times 3$ pixels centered at $x$. For more details, or other applications, see $[13,18]$.

This example was built with the aim to highlight a useful link between the abstract theory of the interval-valued multifunction and the concrete application to image processing. One of the crucial tool in the above set-valued theory is provided by the Hausdorff distance between sets. This special metric plays an important role in the context of digital image processing, where it is used, for example, 
in order to measure the accuracy of certain class of algorithms, such as those of edge detection, already mentioned in the previous list of possible applications. More precisely, if $A$ is the region of interest (ROI) of a given image and $B$ is the corresponding approximation of the ROI $A$ detected by a suitable edge detection algorithm, the Hausdorff distance measure the displacement between $A$ and $B$, in fact evaluating the accuracy (i.e., the approximation error) of the method. For instance, in [55] the Hausdorff distance has been used in order to evaluate the degree of accuracy of an algorithm for the detection of the pervious area of the aorta artery from CT images without contrast medium. This procedure is useful, for example, in the diagnosis of aneurysms of the abdominal aorta artery, especially for patients with severe kidneys pathology for which CT images with contrast medium can not be performed. A similar use of the Hausdorff distance could be done for the edge detection algorithms considered in $[13,18]$.

\section{Conclusions}

A Riemann Lebesgue integral is defined for interval-valued multifunction with respect to interval-valued multisubmeasures. Properties of the integral are established showing in particular that the multimeasure generated is finitely additive. Sufficient conditions for the monotonicity, the order continuity, bounded variation and convergence results are also obtained. A comparison with other integrals is sketchced; an example of an applications in image processing is given highlighting that the advantage of using the notion of interval-valued multifunction in signal analysis is that this formalism allows to include in a unique framework possible uncertainty or the noise on the evaluation of an image at any given pixel. In a future research we will generalize these results in the setting of Banach lattices and we will compare this method with other DIP (digital image processing) algorithms.

Author Contributions: Writing, review and editing, D.C., A.C., A.G., A.I. and A.R.S. All authors have read and agreed to the published version of the manuscript.

Funding: The first and the last authors are members of the working group "Teoria dell'Approssimazione e Applicazioni" of the Italian Mathematical Union (U.M.I.) and they were partially supported by: Grant "Analisi reale, teoria della misura ed approssimazione per la ricostruzione di immagini" (2020) of GNAMPA - INDAM (Italy) and University of Perugia-Fondo Ricerca di Base 2019.

Conflicts of Interest: The authors declare no conflict of interest.

Copyright of the Pictures: The Figure 1 (baboon) are adapted from the original image contained in the repository http:/ / calvados.di.unipi.it/dokuwiki/lib/exe/detail.php?id=ffnamespace:apps\&media=ffnamespace: baboon.jpg under the following license: CC Attribution-Share Alike 3.0 Unported.

\section{References}

1. Moore, R.E. Interval Analysis; Prentice Hall: Englewood Cliffs, NJ, USA, 1966.

2. Weichselberger, $\mathrm{K}$. The theory of interval-probability as a unifying concept for uncertainty. Int. J. Approx. Reason. 2000, 24, 149-170. [CrossRef]

3. Pap, E. Pseudo-additive measures and their applications. In Handbook of Measure Theory, II; Pap, E., Ed.; Elsevier: Amsterdam, The Netherlands, 2002; pp. 1403-1465.

4. Guo, C.; Zhang, D. On set-valued fuzzy measures. Inform. Sci. 2004, 160, 13-25. [CrossRef]

5. El-Owny, H.; Elaraby, A. Improved Generalized Interval Arithmetic. Int. J. Math. Comput. 2013, 19, 84-93.

6. Dempster, A.P. Upper and lower probabilities induced by a multivalued mapping. Ann. Math.Statist. 1967, 38, 325-339. [CrossRef]

7. Zadeh, L.A. Probability measures of fuzzy events. J. Math. Anal. Appl. 1968, 23, 421-427. [CrossRef]

8. Shafer, G. A Mathematical Theory of Evidence; Princeton University Press: Princeton, NY, USA, 1976.

9. Torra, V. Use and Applications of Non-Additive Measures and Integrals. In Non-Additive Measures, Theory and Applications; Torra, V., Narukawa, Y., Sugeno, M., Eds.; Studies in Fuzziness and Soft Computing; Springer: Berlin/Heidelberg, Germany, 2014; Volume 310, pp. 1-33. [CrossRef] 
10. Torra, V.; Narukawa, Y.; Sugeno, M. (Eds.) Non-Additive Measures: Theory and Applications; Studies in Fuzziness and Soft Computing; Springer: Berlin/Heidelberg, Germany, 2014; Volume 310. [CrossRef]

11. Coletti, G.; Petturiti, D.; Vantaggi, B. Models for pessimistic or optimistic decisions under different uncertain scenarios. Int. J. Approx. Reason. 2019, 105, 305-326. [CrossRef]

12. La Torre, D.; Mendivil, F. Minkowski-additive multimeasures, monotonicity and self-similarity. Image Anal. Stereol. 2011, 30, 135-142. [CrossRef]

13. Lopez-Molina, C.; De Baets, B.; Barrenechea, E.; Bustince, H. Edge detection on interval-valued images. In Eurofuse 2011. Advances in Intelligent and Soft Computing; Melo-Pinto, P., Couto, P., Serôdio, C., Fodor, J., De Baets, B., Eds.; Springer: Berlin/Heidelberg, Germany, 2011; Volume 107. [CrossRef]

14. Vrscay, E.R. A generalized class of fractal-wavelet transforms for image representation and compression. Can. J. Elect. Comp. Eng. 1998, 23, 69-84. [CrossRef]

15. La Torre, D.; Mendivil, F.; Vrscay, E.R. Iterated function systems on multifunctions. In Math. Everywhere; Springer: Berlin/Heidelberg, Germany, 2007; pp. 125-138.

16. Wohlberg, B.; De Jager, G. A review of the fractal image coding literature. IEEE Trans. Image Process. 1999, 8 , 1716-1729. [CrossRef]

17. Zhou, Y.; Zhang, C.; Zhang, Z. An efficient fractal image coding algorithm using unified feature and DCT. Chaos Solitons Fractals 2009, 39, 1823-1830. [CrossRef]

18. Jurio, A.; Paternain, D.; Lopez-Molina, C.; Bustince, H.; RMesiar, R.; Beliakov, G. A Construction Method of Interval-Valued Fuzzy Sets for Image Processing. In Proceedings of the 2011 IEEE Symposium on Advances in Type-2 Fuzzy Logic Systems (T2FUZZ), Paris, France, 11-15 April 2011.

19. Birkhoff, G. Integration of functions with values in a Banach space. Trans. Amer. Math. Soc. 1935, 38, 357-378. [CrossRef]

20. Cascales, B.; Rodríguez, J. Birkhoff integral for multi-valued functions. J. Math. Anal. Appl. 2004, 297, 540-560. [CrossRef]

21. Cascales, B.; Rodríguez, J. The Birkhoff integral and the property of Bourgain. Math. Ann. 2005, 331, 259-279. [CrossRef]

22. Marraffa, V. A Birkhoff Type Integral and the Bourgain Property in a Locally Convex Space. Real Anal. Exch. 2007, 32, 409-428. [CrossRef]

23. Potyrala, M. Some remarks about Birkhoff and Riemann-Lebesgue integrability of vector valued functions. Tatra Mt. Math. Publ. 2007, 35, 97-106.

24. Boccuto, A.; Candeloro, D. Integral and ideals in Riesz spaces. Inf. Sci. 2009, 179, 2891-2902. [CrossRef]

25. Candeloro, D.; Sambucini, A.R. Order-type Henstock and McShane integrals in Banach lattices setting. In Proceedings of the 2014 IEEE 12th International Symposium on Intelligent Systems and Informatics (SISY), Subotica, Serbia, 11-13 September 2014; pp. 55-59. [CrossRef]

26. Candeloro, D.; Sambucini, A.R. Comparison between some norm and order gauge integrals in banach lattices. Panam. Math. J. 2015, 25, 1-16.

27. Croitoru, A.; Gavriluţ, A. Comparison between Birkhoff integral and Gould integral. Mediterr. J. Math. 2015, 12, 329-347. [CrossRef]

28. Candeloro, D.; Croitoru, A.; Gavrilut, A.; Sambucini, A.R. An extension of the Birkhoff integrability for multifunctions. Mediterranean J. Math. 2016, 13, 2551-2575. [CrossRef]

29. Candeloro, D.; Di Piazza, L.; Musiał, K.; Sambucini, A.R. Gauge integrals and selections of weakly compact valued multifunctions. J. Math. Anal. Appl. 2016, 441, 293-308. [CrossRef]

30. Caponetti, D.; Marraffa, V.; Naralenkov, K. On the integration of Riemann-measurable vector-valued functions. Monatsh. Math. 2017, 182, 513-536. [CrossRef]

31. Candeloro, D.; Di Piazza, L.; Musiał, K.; Sambucini, A.R. Some new results on integration for multifunction. Ric. Mat. 2018, 67, 361-372. [CrossRef]

32. Candeloro, D.; Sambucini, A.R. A Girsanov result through Birkhoff integral. In International Conference on Computational Science and Its Applications; Springer International Publishing AG: Cham, Switzerland, 2018, Volume LNCS 10960, pp. 676-683. [CrossRef]

33. Gal, S.G. On a Choquet-Stieltjes type integral on intervals. Math. Slov. 2019, 69, 801-814. [CrossRef]

34. Candeloro, D.; Croitoru, A.; Gavrilut, A.; Iosif, A.; Sambucini, A.R. Properties of the Riemann-Lebesgue integrability in the non-additive case. Rend. Circ. Mat. Palermo Ser. 2 2020, 69, 577-589. [CrossRef] 
35. Candeloro, D.; Di Piazza, L.; Musiał, K.; Sambucini, A.R. Multi-integrals of finite variation. Boll. dell'Unione Mat. Ital. 2020, 13, 459-468. [CrossRef]

36. Croitoru, A.; Gavriluţ, A. Convergence results in Birkhoff weak integrability. Boll. Dell'Unione Mat. Ital. 2020, 13, 477-485. [CrossRef]

37. Di Piazza, L.; Musiał, K. Decompositions of Weakly Compact Valued Integrable Multifunctions. Mathematics 2020, 8, 863. [CrossRef]

38. Kadets, V.M.; Tseytlin, L.M. On integration of non-integrable vector-valued functions. Mat. Fiz. Anal. Geom. 2000, 7, 49-65.

39. Kadets, V.M.; Shumyatskiy, B.; Shvidkoy, R.; Tseytlin, L.M.; Zheltukhin, K. Some remarks on vector-valued integration. Mat. Fiz. Anal. Geom. 2002, 9, 48-65.

40. Gavriluţ, A. Remarks of monotone interval valued set multifunctions. Inf. Sci. 2014, 259, 225-230. [CrossRef]

41. Iosif, A.; Gavriluţ, A. Integrability in interval-valued (set) multifunctions setting. Bul. Inst. Politehnic din Iaşi 2017, 63, 65-79.

42. Candeloro, D.; Mesiar, R.; Sambucini, A.R. A special class of fuzzy measures: Choquet integral and applications. Fuzzy Sets Syst. 2019, 355, 83-99. [CrossRef]

43. Pap, E.; Iosif, A.; Gavriluţ, A. Integrability of an Interval-valued Multifunction with respect to an Interval-valued Set Multifunction. Iran. J. Fuzzy Syst. 2018, 15, 47-63.

44. Sambucini, A.R. The Choquet integral with respect to fuzzy measures and applications. Math. Slov. 2017, 67, 1427-1450. [CrossRef]

45. Stamate, C.; Croitoru, A. The general Pettis-Sugeno integral of vector multifunctions relative to a vector fuzzy multimeasure. Fuzzy Sets Syst. 2017, 327, 123-136. [CrossRef]

46. Torra, V.; Narukawa, Y. On network analysis using non-additive integrals: Extending the game-theoretic network centrality. Soft Comput. 2019, 23, 2321-2329. [CrossRef]

47. Drewnowski, L. Topological rings of sets, continuous set functions, integration, I, II, III. Bull. Acad. Polon. Sci. Ser. Math. Astron. Phys. 1972, 20, 277-286.

48. Hu, S.; Papageorgiou, N.S. Handbook of Multivalued Analysis I and II. In Mathematics and Its Applications, 419; Kluwer Academic Publisher: Dordrecht, The Netherlands, 1997.

49. Román-Flores, H.; Chalco-Cano, Y.; Lodwick, W.A. Some integral inequalities for interval-valued functions, Comp. Appl. Math. 2018, 37, 1306-1318.

50. Gavriluţ, A.; Petcu A. Some properties of the Gould type integral with respect to a submeasure. Bul. Inst. Polit. Iasi Sec. Mat. Mec. Teor. Fiz. 2007, 53, 121-130.

51. Labuschagne, C.C.A.; Pinchuck, A.L.; van Alten, C.J. A vector lattice version of Rådström's embedding theorem. Quaest. Math. 2007, 30, 285-308. [CrossRef]

52. Butzer, P.L.; Stens, R.L. Linear Prediction by samples from the past. In Advanced Topics in Shannon Sampling and Interpolation Theory; Springer: New York, NY, USA, 1993; pp. 157-183.

53. Balakrishnan, A. On the problem of time jitter in sampling. IRE Trans. Inf. Theory 1962, 8, 226-236. [CrossRef]

54. Mendivil, F.; Vrscay, E.R. Correspondence between fractal-wavelet transforms and iterated function systems with grey-level maps. In Fractals in Engineering: From Theory to Industrial Applications; Levy-Vehel, J., Lutton, E., Tricot, C., Eds.; Springer: London, UK, 1997.

55. Costarelli, D.; Seracini, M.; Vinti, G. A segmentation procedure of the pervious area of the aorta artery from CT images without contrast medium. Math. Methods Appl. Sci. 2020, 43, 114-133. [CrossRef]

Publisher's Note: MDPI stays neutral with regard to jurisdictional claims in published maps and institutional affiliations.

(C) 2020 by the authors. Licensee MDPI, Basel, Switzerland. This article is an open access article distributed under the terms and conditions of the Creative Commons Attribution (CC BY) license (http://creativecommons.org/licenses/by/4.0/). 